\title{
Headache with Midline Shift: An Uncommon Presentation of Sarcoidosis
}

\author{
Jennifer Y. Wang, MD and David F. Jacobson, MD, FACP \\ Department of Internal Medicine, Santa Clara Valley Medical Center, San Jose, CA, USA.
}

J Gen Intern Med 32(3):363-4

DOI: $10.1007 /$ s11606-016-3807-y

(C) Society of General Internal Medicine 2016

\begin{abstract}
A 51-year-old African American woman presented with 6 months of progressively worsening left frontal headaches, 1 week of blurred vision in her left eye, and 1 day of nausea and vomiting. She denied other neurologic or respiratory symptoms.

Examination revealed decreased visual acuity and a mild afferent pupillary defect in the left eye. MRI of the brain revealed a dural-based mass centered on the left sphenoid wing with significant vasogenic edema and midline shift (Fig. 1). Computed tomography of the chest demonstrated multiple opacities in the upper and mid-lung fields and bilateral mediastinal and hilar lymphadenopathy. Further workup was negative, and biopsy of the brain lesion revealed noncaseating granulomatous inflammation consistent with sarcoidosis.

Neurologic complications occur in only $5-10 \%$ of sarcoidosis cases, and may not be associated with other systemic manifestations. ${ }^{1}$ The most common presenting symptom is cranial neuropathy, most frequently of the facial or optic nerve. ${ }^{1}$ Leptomeningeal disease accounts for $10-20 \%$ of neurosarcoidosis, and can manifest as aseptic meningitis or a dural mass lesion, as in this patient. ${ }^{2}$
\end{abstract}

Received May 24, 2016

Revised June 22, 2016

Accepted June 28, 2016

Published online July 18, 2016
Our patient improved rapidly with corticosteroids (Fig. 2), which is the first-line therapy for neurosarcoidosis. Combination therapy with TNF-alpha antagonists and steroid-sparing agents may be considered in refractory or severely disabling cases. $^{1,2}$

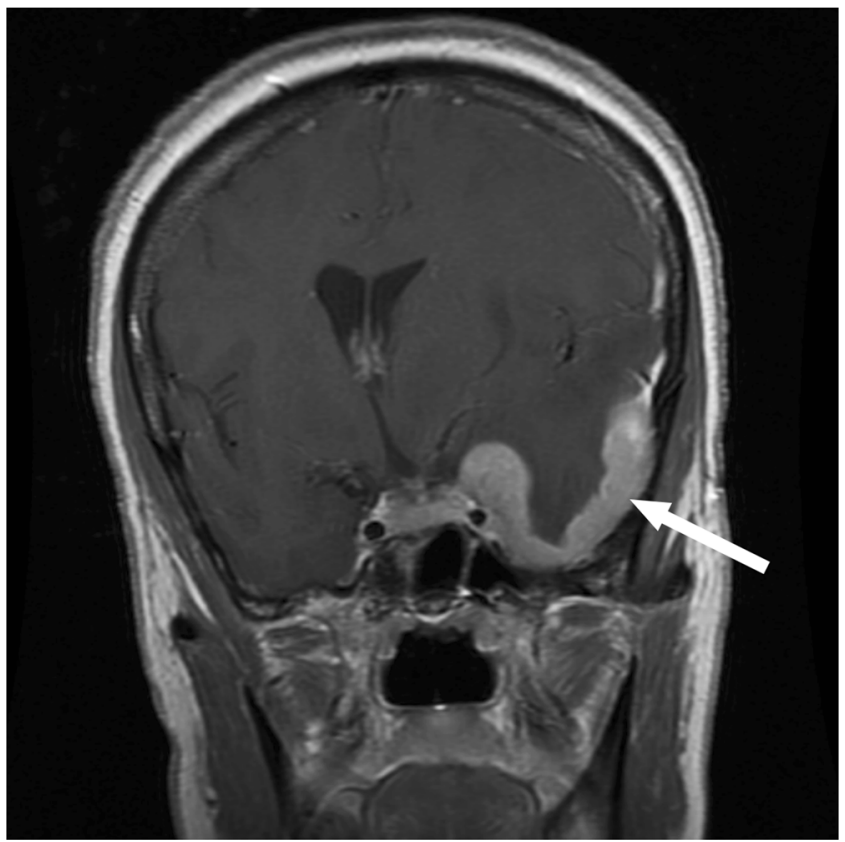

Figure 1 Coronal T1-weighted MRI scan of the brain with gadolinium enhancement, initial presentation. Arrow indicates dural-based mass over the left sphenoid wing. 


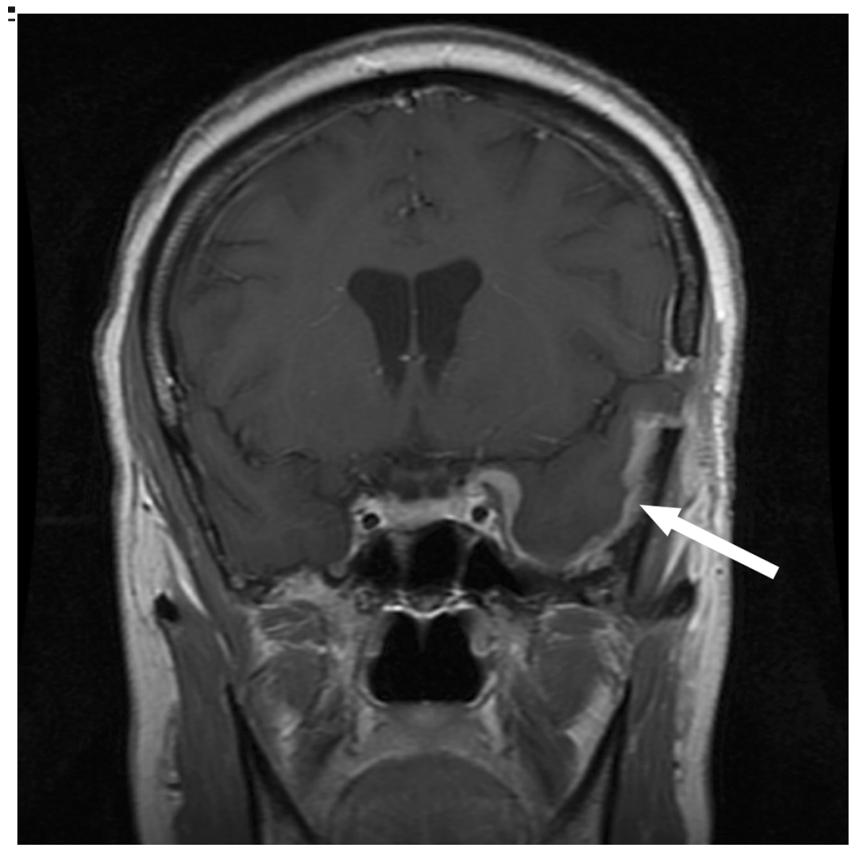

Figure 2 Coronal T1-weighted MRI scan of the brain with gadolinium enhancement, status post 4 weeks of high-dose corticosteroid therapy. Significant decrease in size of the dural-based mass (arrow) and resolution of midline shift are noted.
Corresponding Author: David F. Jacobson, MD, FACP; Department of Internal MedicineSanta Clara Valley Medical Center, 751 South Bascom Avenue, San Jose, CA 95128, USA (e-mail: david.jacobson@hhs.sccgov.org).

\section{Compliance with Ethical Standards:}

Conflict of Interest: The authors declare that they have no conflict of interest.

\section{REFERENCES}

1. Tavee JO, Stern BJ. Neurosarcoidosis. Clin Chest Med. 2015;36(4):643-656.

2. Krumholz A, Stern BJ. Neurologic manifestations of sarcoidosis. Handb Clin Neurol. 2014;119:305-333. 